\title{
Variación estacional de las amputaciones en un hospital universitario de Monterrey, México (2009-2012)
}

\section{Seasonality variation of amputations in a university hospital from Monterrey, Mexico} (2009-2012)

\author{
Sandra Castillo-Guzmán ${ }^{1 *}$, Dionicio Palacios-Ríos ${ }^{1}$, Norma G. López-Cabrera1, \\ Marco A. Hernández-Guedea², Nora H. Rodríguez-Rodriguez ${ }^{1}$ y Omar González-Santiago 3 \\ ${ }^{1}$ Servicio de Anestesiología; ${ }^{2}$ Servicio de Cirugía General. Hospital Universitario Dr. José E. González; ${ }^{3}$ Posgrado en Farmacia, Facultad de Ciencias \\ Químicas, Universidad Autónoma de Nuevo León. Monterrey, Nuevo León, México
}

\section{Resumen}

Antecedentes: Una de las principales complicaciones de la diabetes mellitus es la amputación de alguna extremidad. En todo el mundo, la prevalencia de amputaciones asociadas a la diabetes es muy variada y tiene un impacto considerable en la calidad de vida del paciente. Objetivo: Analizar la frecuencia de las amputaciones en el Hospital Universitario Dr. José E. González y evaluar si se presenta un patrón estacional. Método: Se realizó un estudio retrospectivo de 2009 a 2012 , en el que se revisaron los expedientes de pacientes diabéticos que se sometieron a amputación. La estacionalidad se analizó con la bondad de ajuste de ji al cuadrado. Resultados: Se analizaron 456 amputaciones. Los resultados muestran que febrero es el mes que presenta la mayor frecuencia de amputaciones. La estación anual con mayor número de amputaciones fue el invierno. Conclusiones: Las amputaciones en pacientes diabéticos del área metropolitana de Monterrey presentan un patrón estacional, siendo los meses de invierno los de mayor frecuencia.

PALABRAS CLAVE: Diabetes. Amputaciones. Estacionalidad. Invierno. México.

\begin{abstract}
Background: The amputation of an extremity is a main complication of Diabetes mellitus. Worldwide the prevalence of amputations associated with diabetes mellitus is variable and had a considerable impact in the quality of life. Objective: Analyze the frequency of amputations in the University Hospital, Dr José E González and evaluate if a seasonal pattern is present. Method: A retrospective analysis from 2009 to 2012 was carried out. Clinical files of diabetic patients undergoing to amputation were studied. The seasonality was evaluated with a chi square goodness of fit. Results: A total of 456 amputations were studied. Results shown that February was the month with highest frequency of amputations while winter was the annual season with highest frequency of amputations. Conclusions: Amputations of diabetic patients from Metropolitan Monterrey Mexico show a seasonal pattern being the winter months those that present highest frequency.
\end{abstract}

KEY WORDS: Diabetes. Amputations. Seasonality. Winter. Mexico. 


\section{Introducción}

La diabetes mellitus es una enfermedad de proporciones epidémicas que ha duplicado su incidencia en todo el mundo en los últimos 10 años. Según datos de la Organización Mundial de la Salud, se estima que 422 millones de adultos padecían diabetes en el año 2014, y fue la causa de 1.5 millones de defunciones en el año 2012'. Las complicaciones de esta enfermedad afectan a muchas partes del cuerpo, y en términos generales incrementan la mortalidad en quienes la padecen. Dentro de las complicaciones que se presentan se encuentran las siguientes: insuficiencia renal, infartos al miocardio, accidente cerebrovascular, perdida de la visión, daño a los nervios periféricos y un riesgo aumentado de amputaciones no traumáticas ${ }^{2}$. Dichas complicaciones se asocian a neuropatía, enfermedad vascular periférica, mal control metabólico y deterioro de la inmunidad. Todos estos elementos pueden conducir, en el caso de las complicaciones del pie, a ulceración, infección y eventualmente amputación. La variación estacional de las amputaciones no está bien descrita.

La amputación de alguna extremidad inferior es una de las complicaciones más temidas por los pacientes con diabetes y está asociada con una pobre calidad de vida. La incidencia de amputaciones en pacientes con diabetes es muy variada: se ha estimado una tasa global que va de 46.1 a 9600 amputaciones por 100,000 habitantes, valor muy alto si se compara con la incidencia de amputaciones en la población general, que es de 5.8 a 31 amputaciones por 100,000 habitantes. Comparados con la población general, los pacientes tienen un riesgo 30 veces superior de tener una amputación no traumática ${ }^{3}$. Se considera que en los países en vías de desarrollo la amputación implica la ruina financiera para la familia del paciente $e^{4,5}$.

Dado el impacto considerable que tienen las amputaciones, el objetivo de este estudio fue describir de forma mensual y por estación anual la frecuencia de amputaciones no traumáticas de miembros inferiores en pacientes con diabetes mellitus.

\section{Método}

El número de amputaciones en pacientes con diabetes, así como los datos sociodemográficos, se obtuvieron del archivo general del Hospital Universitario Dr. José E. González, de la Universidad Autónoma de
Nuevo León. El periodo de estudio fue de enero de 2009 a diciembre de 2012.

El número de amputaciones mensuales y por estación del año fue ajustado a 30 y 90 días, respectivamente. Las amputaciones se agruparon mensualmente y por estación del año según el sexo, la edad (>60 o $<60$ años), la escolaridad (analfabeto, primaria, secundaria y preparatoria-universidad), el estado civil (soltero-separado, casado y viudo) y el tipo de cirugía (uno o más ortejos, supramaleolar, supracondílea y transtibial). El análisis elemental es observacional, descriptivo y retrospectivo. La diferencia mensual y estacional según los diferentes grupos se analizó con la prueba de bondad de ajuste de ji al cuadrado. El riesgo de amputación en invierno respecto al verano se analizó con una regresión logística y se expresó como odds ratio $(\mathrm{OR})$.

El estudio fue aprobado por el Comité de Ética de la Facultad de Medicina de la Universidad Autónoma de Nuevo León; el número de registro es AN14-002.

\section{Resultados}

\section{Características de la población}

Durante el periodo de estudio se realizaron 456 amputaciones no traumáticas en pacientes con diabetes mellitus. El principal sitio de amputación fue supracondílea $(50.4 \%)$, mientras que el menor fue en uno o más ortejos (18\%). El mayor porcentaje de amputaciones se observó en los hombres (67.8\%), en personas mayores de 60 años (48.8\%), con escolaridad primaria $(56.8 \%)$ y casados (61\%). Con excepción de la edad, en el resto de los grupos se observó una diferencia significativa $(p<0.05)$ (Tabla 1).

\section{Amputaciones mensuales}

Según el mes, los mayores porcentajes de amputaciones ocurrieron en enero y en febrero (9.6 y $13.4 \%$, respectivamente), mientras que los menores se observaron en junio y julio (6.1 y $7.7 \%$, respectivamente). La diferencia mensual fue estadísticamente significativa en el sexo femenino $(p=0.04)$, las personas mayores de 60 años $(p=0.03)$ y los casados $(p<0.01)$ (Tabla 2)

\section{Amputaciones estacionales}

De forma general se observó que la mayor frecuencia de amputaciones ocurrió en la estación de invierno, y la menor frecuencia en verano. Esta diferencia 
estacional fue estadísticamente significativa solo en los que tenían escolaridad de primaria $(p=0.04)$ y estado civil casado $(p<0.01)$. El resto de los grupos no presentó diferencia significativa (Tabla 3). El riesgo de amputación en invierno respecto al verano fue estadísticamente significativo en las personas con

Tabla 1. Frecuencia de amputaciones en el Hospital Universitario Dr. José E. González (2009-2012)

\begin{tabular}{lcc}
\hline Variable & Frecuencia & $\mathbf{p}$ \\
\hline Sexo & & \\
$\quad$ Masculino & 310 & $<0.01$ \\
Femenino & 148 & \\
Edad (años) & & \\
$<60$ & 234 & \\
$>60$ & 223 & 0.61 \\
Escolaridad & & \\
Analfabeto & 41 & \\
Primaria & 226 & \\
Secundaria & 90 & \\
Preparatoria-universitaria & 41 & $<0.01$ \\
Estado civil & & \\
Casado & 262 & \\
Viudo & 59 & \\
Soltero/divorciado & 109 & $<0.01$ \\
Cirugía & & \\
Uno o más ortejos & 82 & \\
Supramaleolar & 34 & \\
Transtibial & 110 & \\
Supracondílea & 230 & $<0.01$ \\
\hline
\end{tabular}

escolaridad de secundaria $(\mathrm{OR}=3.31$; intervalo de confianza del 95\%: 1.02-10.69).

\section{Discusión}

En este estudio se describe la variación mensual y por estación del año de las amputaciones no traumáticas en pacientes con diabetes mellitus en un hospital de tercer nivel del noreste de México. Varios procesos fisiológicos y patológicos han mostrado variación estacional tanto en personas sanas como en enfermas. Ejemplos de estos son el cortisol, la adrenalina, la glucosa, la presión sanguínea, la frecuencia cardiaca y la Hemoglobina glicosilada $(\mathrm{HbA1c})$, entre otros ${ }^{6}$. Se ha reportado inclusive un patrón estacional en la incidencia de diabetes tipo $2^{7}$.

Al igual que en otros estudios, el porcentaje de amputaciones fue mayor en el sexo masculino que en el femenino $0^{8-10}$. Posibles causas de esta diferencia podrían ser un cuidado deficiente de los pies por parte de los varones, hábitos inadecuados como no apegarse a la dieta adecuada para su enfermedad, y también no tener revisiones periódicas de sus valores de glucosa y lípidos sanguíneos ${ }^{11,12}$.

En este estudio se observó una diferencia significativa en la frecuencia de amputaciones mensuales y por estación anual. A la fecha son pocos los estudios que analizan la frecuencia de amputaciones desde una perspectiva temporal en los pacientes con

Tabla 2. Número de amputaciones según el mes

\begin{tabular}{|c|c|c|c|c|c|c|c|c|c|c|c|c|c|}
\hline Variable & Enero & Febrero & Marzo & Abril & Mayo & Junio & Julio & Agosto & Septiembre & e Octubre & Noviembre & Diciembre & $p$ \\
\hline \multicolumn{14}{|l|}{ Sexo } \\
\hline Femenino & 15 & 25 & 9 & 13 & 17 & 8 & 12 & 9 & 13 & 11 & 6 & 10 & 0.02 \\
\hline Masculino & 27 & 41 & 31 & 28 & 19 & 20 & 22 & 30 & 27 & 22 & 22 & 17 & 0.06 \\
\hline \multicolumn{14}{|l|}{ Edad (años) } \\
\hline$<60$ & 16 & 36 & 16 & 17 & 17 & 18 & 20 & 21 & 21 & 16 & 15 & 16 & 0.06 \\
\hline$>60$ & 26 & 29 & 23 & 24 & 19 & 10 & 14 & 17 & 19 & 16 & 12 & 11 & 0.02 \\
\hline \multicolumn{14}{|l|}{ Escolaridad } \\
\hline Analfabeto & 4 & 9 & 3 & 4 & 4 & 4 & 3 & 1 & 3 & 4 & 2 & 1 & 0.27 \\
\hline Primaria & 28 & 26 & 20 & 18 & 17 & 14 & 15 & 19 & 24 & 15 & 13 & 13 & 0.16 \\
\hline Secundaria & 4 & 15 & 5 & 9 & 9 & 5 & 9 & 10 & 8 & 6 & 5 & 6 & 0.24 \\
\hline Preparatoria-universitaria & 6 & 9 & 4 & 3 & 1 & 2 & 3 & 4 & 1 & 4 & 2 & 3 & 0.15 \\
\hline \multicolumn{14}{|l|}{ Estado civil } \\
\hline Casado & 29 & 44 & 22 & 19 & 23 & 19 & 17 & 23 & 25 & 15 & 16 & 7 & 0.001 \\
\hline Viudo & 5 & 10 & 4 & 10 & 5 & 3 & 2 & 4 & 2 & 7 & 3 & 5 & 0.13 \\
\hline Soltero/divorciado & 9 & 10 & 10 & 10 & 7 & 5 & 12 & 10 & 11 & 8 & 6 & 12 & 0.87 \\
\hline \multicolumn{14}{|l|}{ Cirugía } \\
\hline Uno o más ortejos & 9 & 11 & 7 & 4 & 6 & 7 & 3 & 6 & 5 & 13 & 4 & 8 & 0.23 \\
\hline Supramaleolar & 1 & 5 & 4 & 1 & 3 & 3 & 4 & 3 & 1 & 2 & 3 & 4 & 0.8 \\
\hline Supracondílea & 25 & 30 & 22 & 24 & 23 & 13 & 13 & 20 & 21 & 13 & 12 & 12 & 0.02 \\
\hline Transtibial & 10 & 14 & 5 & 12 & 5 & 6 & 13 & 9 & 12 & 8 & 11 & 6 & 0.7 \\
\hline
\end{tabular}


Tabla 3. Número de amputaciones según la estación del año

\begin{tabular}{lccccc}
\hline & Primavera Verano & Otoño & Invierno & $\mathbf{p}$ \\
\hline Sexo & & & & & \\
$\quad$ Femenino & 39 & 32 & 27 & 48 & 0.08 \\
Masculino & 71 & 77 & 65 & 93 & 0.13 \\
& & & & & \\
Edad (años) & 51 & 61 & 48 & 70 & 0.16 \\
$<60$ & 58 & 47 & 44 & 71 & 0.04 \\
$>60$ & & & & & \\
Escolaridad & & & & & \\
Analfabeto & 14 & 5 & 9 & 13 & 0.18 \\
Primaria & 48 & 57 & 44 & 74 & 0.02 \\
Secundaria & 22 & 27 & 17 & 22 & 0.52 \\
Preparatoria-universitaria & 8 & 9 & 7 & 17 & 0.11 \\
Estado civil & & & & & \\
Casado & & & & & \\
Viudo & 63 & 64 & 45 & 86 & 0.001 \\
Soltero/divorciado & 16 & 10 & 12 & 20 & 0.25 \\
Cirugía & 23 & 29 & 27 & 28 & 0.86 \\
$\quad$ Uno o más ortejos & & & & & \\
Supramaleolar & 15 & 12 & 20 & 23 & 0.24 \\
Supracondílea & 3 & 6 & 4 & 8 & 0.42 \\
Transtibial & 61 & 50 & 35 & 69 & 0.007 \\
\hline & 19 & 27 & 25 & 25 & 0.68 \\
\hline
\end{tabular}

diabetes mellitus. Aun cuando se reporta una diferencia según la estación del año, hay contradicción en cuanto a la estación en la que ocurre el mayor número de amputaciones. Un estudio en Nueva York reportó que la mayor frecuencia de amputaciones se producía en primavera, lo cual difiere de nuestros hallazgos ${ }^{13}$. Por otro lado, un estudio en Hong Kong encontró una asociación positiva entre la frecuencia de amputaciones y la temperatura ambiental, lo cual sugiere que los meses calurosos se asocian con mayor número de amputaciones; sin embargo, los autores consideran que la alta humedad propia del país podría enmascarar los efectos de la temperatura ${ }^{14}$. Otro estudio en Pakistán reportó la mayor frecuencia de amputaciones en invierno, lo cual concuerda con nuestro estudio ${ }^{15}$.

Varios factores pueden influir en la variación estacional de las amputaciones; dentro de estos, la temperatura y el grado de humedad podrían considerarse como dos factores importantes. La exposición a temperaturas frías podría afectar la perfusión sanguínea de las extremidades de los pacientes, y la humedad alta puede favorecer las condiciones para la infección de dichas extremidades. Sin embargo, hasta el momento la mayoría de las investigaciones no explican con precisión las causas o los factores de dicha variación estacional. Se necesitan más estudios al respecto que tomen en cuenta otras variables, como el control metabólico, ya que, como se sabe, este factor influye de manera importante en el riesgo de amputación. Cabe mencionar que, en México, el 70\% de los pacientes no tienen un buen control metabólico y muchos de ellos no tienen una revisión adecuada de sus pies ${ }^{16}$.

Conocer la variación estacional de las amputaciones podría ayudar a los tomadores de decisiones a diseñar estrategias preventivas, especialmente en la estación de invierno del área metropolitana de Monterrey. Programar visitas antes y durante los meses de invierno, y aconsejar una mayor inspección de los pies a los pacientes durante esta época, podrían contribuir a una mejora sustancial.

Finalmente, deben considerarse las limitaciones de este estudio. Entre ellas podemos mencionar que nuestros resultados tal vez no apliquen para otras ciudades de México o del mundo, por lo que se recomienda prudencia en la interpretación. Además, en nuestro estudio no se incluyó el análisis de variables meteorológicas como la temperatura y la humedad. No se contó con los datos de control metabólico, como la $\mathrm{HbA} 1 \mathrm{c}$, ni con las condiciones de prestación de servicio, como Instituto Mexicano del Seguro Social, salubridad u otras instituciones. Todos esos factores bien podrían influir también en la variación estacional de las amputaciones.

\section{Conclusiones}

La frecuencia de las amputaciones en los pacientes diabéticos del área metropolitana de Monterrey presenta un patrón estacional, siendo los meses de invierno los de mayor número de amputaciones.

\section{Agradecimientos}

A Ramiro A. Torres Villarreal, Asahel Alonso Alanís y Teresa A. Nava Obregón, por el apoyo técnico.

\section{Conflicto de intereses}

Los autores declaran que no tienen conflictos de intereses.

\section{Responsabilidades éticas}

El estudio fue aprobado por el Comité de Ética de la Facultad de Medicina de la Universidad Autónoma de Nuevo León. El número de registro es AN14-002.

Consentimiento informado: dado que el estudio es de naturaleza retrospectiva, no se solicitó el consentimiento informado. 


\section{Bibliografía}

1. Roglic G. Global report on diabetes. Geneva, Switzerland: World Health Organization; 2016.

2. Forbes JM, Cooper ME. Mechanisms of diabetic complications. Physiol Rev. 2013;93:137-88

3. Moxey PW, Gogalniceanu P, Hinchliffe RJ, Loftus IM, Jones KJ, Thompson MM, et al. Lower extremity amputations - a review of global variability in incidence. Diabet Med. 2011;28:1144-53.

4. Driver VR, Fabbi M, Lavery LA, Gibbons G. The costs of diabetic foot: the economic case for the limb salvage team. J Vasc Surg. 2010;52(3 Suppl):17S-22S.

5. Kerr M, Rayman G, Jeffcoate WJ. Cost of diabetic foot disease to the National Health Service in England. Diabet Med. 2014;31:1498-504.

6. Tseng C-L, Brimacombe M, Xie M, Rajan M, Wang H, Kolassa J, et al. Seasonal patterns in monthly hemoglobin $\mathrm{A} 1 \mathrm{c}$ values. Am J Epidemiol. 2005:161:565-74.

7. Doró $P$, Benko R, Matuz M, Soós G. Seasonality in the incidence of type 2 diabetes: a population-based study. Diabetes Care. 2006;29:173.

8. Ebskov LB. Epidemiology of lower limb amputations in diabetics in Denmark (1980 to 1989). Int Orthop. 1991;15:285-8.

9. Trautner C, Haastert B, Mauckner P, Gätcke L-M, Giani G. Reduced incidence of lower-limb amputations in the diabetic population of a Ger- man city, 1990-2005: results of the Leverkusen Amputation Reduction Study (LARS). Diabetes Care. 2007;30:2633-7.

10. Veresiu IA, lancu SS, Bondor C. Trends in diabetes-related lower extremities amputations in Romania - a five year nationwide evaluation. Diabetes Res Clin Pract. 2015;109:293-8.

11. Rossaneis MA, Haddad M do CFL, Mathias TA de F, Marcon SS. Differences in foot self-care and lifestyle between men and women with diabetes mellitus. Rev Lat Am Enfermagem. 2016;24:e2761.

12. Mathew R, Gucciardi E, De Melo M, Barata P. Self-management experiences among men and women with type 2 diabetes mellitus: a qualitative analysis. BMC Fam Pract. 2012;13:122.

13. Armstrong DG, Lavery LA, van Houtum WH, Harkless LB. Seasonal variations in lower extremity amputation. J Foot Ankle Surg. 1997;36:146-50.

14. Leung HB, Ho YC, Wong WC, Guerin J. Seasonal variations in non-traumatic major lower limb amputation in Hong Kong Chinese diabetic patients. Hong Kong Med J. 2007;13:379-81.

15. Ateeq S, Mehmood R. Seasonal variations and hospitalization due to diabetic foot complications. JRMC. 2013;17:201-3. Disponible en: https:// www.journalrmc.com/volumes/1395217808.pdf

16. Vargas-Daza ER, Rivera-Pérez MDP, Villarreal-Ríos E, Galicia-Rodríguez L, Martínez-González L. Exploración física y morbilidad de miembros pélvicos en el paciente diabético tipo 2. Rev Med Inst Mex Seguro Soc. 2013;51:314-9 